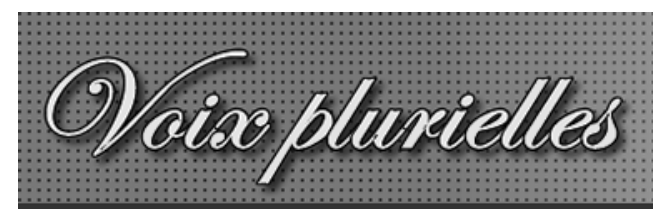

Revue de l'Association des Professeur-e-s de Français des Universités et Collèges Canadiens

\author{
Voix plurielles \\ Volume 3, Numéro 1 : mai 2006
}

Suzanne Crosta

\title{
Postface à Voix plurielles 3.1
}

Citation MLA : Crosta, Suzanne. «Postface à Voix plurielles 3.1.»Voix plurielles 3.1 (mai 2006).

(c) Voix plurielles, revue électronique de l'APFUCC 2006. 


\title{
Postface à Voix plurielles 3.1
}

\author{
Suzanne Crosta \\ Université McMaster
}

Mai 2006

Q uelle belle tâche d'être porte-parole de notre Université pour dire sa joie et son admiration devant la réussite de ces deux jours d'intense communication et de ce volume qui en est l'heureux aboutissement! Pour nous maintenir dans les bornes de la plus stricte vérité et ménager toutes les modesties, tout en faisant miroiter quelques heureux reflets, nous en resterons à la métaphore. Enfermant mon émotion, elle nous guidera dans ce dernier survol des thèmes abordés et dessinés sous tant de variétés. Comme elle suggère plus qu'elle n'exprime, elle ne m'obligera pas à effectuer le long enchaînement, ce qu'on appellerait la concaténation des grandes idées émises : l'image fera office de discret et bienveillant catalyseur pour permettre à chacun(e) de colorer ses propres émotions sous mon clin d'oeil complice.

L'ensemble de l'oeuvre accomplie pendant ce Colloque me fait penser à des floralies, une grande exposition de fleurs de toutes les formes, couleurs et fragrances propres à charmer le regard et à éveiller les sens dans une féerie de perspectives originales. Ce mot de floralies, toujours pluriel, inclut le multiple sous un principe d'unité : l'exposition marque l'unité d'intention, tandis que la variété des fleurs illustre la diversité, par où nous rejoignons l'idée directrice du colloque. Dix-huit exposés ont été soumis à notre oeil intérieur, volontiers critique mais cependant prompt à se laisser charmer par l'harmonie. Notre constante recherche de vérité et de cohérence nous pousse à une critériologie, une critique de notre connaissance lucide et ouverte aux approches nouvelles.

Ce volume dessine un arc-en-ciel de couleurs qui prend d'abord sa source dans la langue elle-même, notre langue française. Premier substrat chargé d'expériences millénaires parfois subtilement paradigmatiques, elle ne résiste pas à inclure des teintes modernes. Elle s'ouvre à l'expression neuve, cependant qu'elle conserve toute une frange bigarrée qui fonde son pouvoir évocateur. Des tonalités et des harmoniques se sont ajoutés qui correspondent aux préoccupations et aux sensibilités de notre époque. Elle est un instrument de plus en plus raffiné, un système ouvert indéfiniment perfectible. Et vous en avez bien usé.

La littérature offre un espace où s'expriment les enthousiasmes et les craintes, les joies et les douleurs, les incompréhensions et les cris de ralliement, les injustices et les générosités. Elle ne dégage pas du creuset où se vivent les transes, mais elle invite au sublime; si elle est oasis par les soifs qu'elle étanche et les fraîcheurs qu'elle offre, elle ne s'arrête pas sur elle-même, soulevant souvent des aspirations plus élevées. Ne pourrait-on pas la dire lieu d'éloquence où se débattent librement, et plus ouvertement qu'en politique, toutes les questions sur l'homme dans ses réalités les plus profondes comme les plus accidentelles, l'urgent côtoyant le long terme, l'aujourd'hui 
un peu narcissique retrouvant nombre de ses traits dans le passé, le voisin embrassant l'éloigné, le mesquin jalousant parfois, et malheureusement, le noble. Bien davantage que scène ou théâtre, lieu d'affrontement ou cercle de privilégiés du verbe, la littérature est parénèse ( $\pi \alpha \rho \alpha \imath \varepsilon \sigma \_\varsigma$ : encouragement), mêlant les analyses aux avertissements, les conseils aux encouragements.

Les fleurs de la globalisation poussent encore à l'ombre, se pointant timidement au soleil. Quelque jardinier expert réussira-t-il à les dé-gêner, à affecter favorablement leurs gênes et à les préserver d'une toxicité inquiétante? C'est un problème de grande urgence. La Francophonie, suivant Bousse Allouche, est de nature à présenter un certain contrepoids pour préserver les richesses linguistiques et culturelles menacées de nivellement ou même d'évincement. Quant aux mouvements migratoires - déplacements massifs ou graduels- il est naturel qu'ils se produisent, et ils se réaliseront toujours naturellement, mais quelle différence si l'on reconnaît qu'une foule n'est pas une communauté, qu'une communauté se caractérise par une structure organisée, que l'organisme doit être informé par des valeurs fondamentales qui en sont l'âme et qui maintiennent la vie. Pour se réaliser en plénitude, cette vie exige un constant effort de volonté qui la protège et l'épanouit : seule la volonté introduit dans l'ordre de l'amour. On parlera alors d'intégration réussie, à la manière d'une greffe heureuse sur un corps social revigoré, le système immunitaire étant rassuré.

Que de belles fleurs dans la littérature francophone, chacune avec ses teintes propres suivant les méridiens qui les ont vues naître, du Maghreb aux autres Pays d'Afrique, de la Macédoine au Liban, des Pays d'Europe à ceux de la Caraïbe et d'Amérique qui les prolongent outre-Atlantique, avec un accent particulier sur quelques Provinces du Canada. Une sympathie ouverte ne porte pas ombrage à une autre sympathie, et la recherche constante d'humanité rend accueillant sans limite et purifie des égoïsmes faciles ou inconsidérés. Nous sommes sensibilisé(e)s à l'absence révoltante de justice dans certains Pays africains, et Tracy Russell s'effraye avec raison de la situation tragique des femmes, victimes de toutes les façons inhumaines.

Enfin, de magnifiques fleurs de tendresse, celles qui s'ouvrent en poésie, poésie de la vie vécue en profondeur, celle qui est au coeur de tout humain ne refusant pas de regarder l'autre et d'y admirer des différences propres à moirer les rapports sociaux. Certaines difficultés du vivre-ensemble apparaissent en France, mais non pas là seulement. Il y a encore des mentalités à changer, des conversions à opérer, un humanisme à redéfinir, un respect plus profond à éveiller, une disponibilité à offrir. Notre temps a peut-être oublié cette distinction fondamentale que les Anciens établissaient entre convaincre et persuader : le premier s'adresse à l'intelligence, à la faculté de raisonner, en montrant les enchaînements logiques, suivant les idées claires de Descartes : les débats qui visent cet objectif poussent rarement à l'action. Par contre, persuader s'adresse non seulement à l'intelligence, mais aussi à la volonté qu'il s'agit de mouvoir vers le bien. Un certain état de grâce est nécessaire à la conduite humaine pour traduire une perfection fondamentale, une bienveillance que le dictionnaire latin traduit tout aussi bien par benevolentia que par humanitas. Voilà clairement proclamé que le destin humain est affaire de volonté, de volonté saine, tournée vers le bien.

En effet, nous avons bien vu et bien entendu la sincérité rassurante des propos et des engagements de nos orateurs, et c'est de bon augure. Ce sont des voix qui crient et qui émeuvent, donc qui réussiront à persuader. Il faut brûler d'un grand feu en soi pour réchauffer les autres et 
les entraîner dans le feu de l'action humanitaire, plus que jamais nécessaire dans les turbulences et les dangereux désordres des temps présents. Volonté, bienveillance, joie, pour un humanisme aux multiples visages, tous souriants.

Notre gratitude va a chacun et à chacune pour sa générosité de temps et d'efforts. Le mérite des auteurs est double: il se traduit par la quête de la perfection dans le travail de recherche et d'organisation de la pensée, mais aussi les auteurs enrichissent notre savoir et stimulent notre propre questionnement, nous les lecteurs, en invitant notre esprit et notre coeur à vibrer au rythme du monde. L'Université McMaster est très fière de cette riche moisson de vos voix plurielles gravées dans ce volume, et vos professeurs vous regardent avec un sourire entendu, tout en sachant que vous êtes en mesure de relever les défis du jardin que vous êtes en train de cultiver.

\author{
Suzanne Crosta \\ Vice-doyenne, Faculté des sciences humaines \\ Université McMaster
}

